\title{
Incidência do carcinoma de células escamosas da pele na cidade de Tubarão (SC) - Brasil nos anos de 2000, 2003 e 2006*
}

\author{
Incidence of squamous cell carcinoma of the skin in the city \\ of Tubarao (SC) - Brazil in the years 2000, 2003, and 2006
}

\author{
Daniel Holthausen Nunes ${ }^{1}$ \\ Ramon Vieira e Silva ${ }^{3}$
}

\author{
Liliane Back $^{2}$ \\ Vitor de Sousa Medeiros ${ }^{4}$
}

\begin{abstract}
Resumo: FunDAMENTOS: Observa-se tendência mundial de aumento na incidência do carcinoma de células escamosas da pele, porém na região sul do estado de Santa Catarina (SC) não há dados sobre a incidência desse tipo de câncer.

OвJETIVOS: Estabelecer dados epidemiológicos do carcinoma de células escamosas da pele em Tubarão (Santa Catarina).

MÉTodos: Laudos anatomopatológicos positivos para o carcinoma de células escamosas da pele dos laboratórios de Tubarão foram revisados quanto às variáveis: ano, idade, gênero, localização, subtipo histológico, maior diâmetro da lesão e comprometimento das margens. A incidência anual foi calculada utilizando o número de neoplasias encontradas e a população anual estimada para os anos de 2000, 2003 e 2006. RESULTADOS: Foi encontrada uma incidência de carcinoma de células escamosas da pele por 100.000 habitantes de 50,86 para o ano de 2000 , de 71,16 para 2003 e de 94,39 para 2006 . Não houve predomínio de gênero, a face foi o local de acometimento mais frequente em ambos os gêneros e o subtipo histológico mais comum foi o bem diferenciado.

CONCLUSÃO: A incidência do carcinoma de células escamosas da pele encontrada superou a estimativa da literatura consultada. Houve aumento na sua incidência; as variáveis idade, localização e tipo histológico foram concordantes com a literatura, porém a distribuição quanto ao gênero se mostrou diferente. Palavras-chave: Carcinoma de células escamosas; Carcinoma de células escamosas /epidemiologia; Incidência; Neoplasias cutâneas
\end{abstract}

\begin{abstract}
BACKGROUND: A worldwide increasing trend in the incidence of squamous cell carcinoma of the skin has been observed but there are no studies regarding the incidence of this cancer in the Southern region of the State of Santa Catarina.

OвJестіves: To establish epidemiological data regarding squamous cell carcinoma of the skin in Tubarao (state of Santa Catarina).

METHODS: Anatomopathological reports, positive for squamous cell carcinoma of the skin, found in laboratories of the city of Tubarao, were analyzed regarding year, age, gender, site, histological subtype, largest diameter, and biopsy margins. The annual incidence was calculated using the number of neoplasias found and the estimated population for the years 2000, 2003, and 2006.

RESULTS: The incidence of squamous cell carcinoma of the skin per 100,000 habitants was 50.86 for the year 2000, 71.16 for 2003 and 94.39 for 2006. There was no gender dominance, the face was the most frequent location affected in both genders, and the most common histological subtype was well differentiated tumor.

CONCLUSION: The detected incidence of squamous cell carcinoma of the skin surpassed the incidence estimated in the literature. There was a considerable increase in its incidence; the variables age and location agreed with information found in the literature but gender distribution differed.

Keywords: Carcinoma, squamous cell; Carcinoma, squamous cell/epidemiology; Incidence; Skin neoplasms
\end{abstract}

Recebido em 14.10.2008.

Aprovado pelo Conselho Consultivo e aceito para publicação em 31.07.09.

* Trabalho realizado na Universidade do Sul de Santa Catarina (Unisul) - Tubarão (SC), Brasil.

Conflito de interesse: Nenhum / Conflict of interest: None

Suporte financeiro: Nenhum / Financial funding: None

Mestre em ciências médicas. Professor do curso de medicina da Universidade do Sul de Santa Catarina e Universidade Federal de Santa Catarina - Tubarão (SC), Brasil

Acadêmica do curso de medicina da Universidade do Sul de Santa Catarina (Unisul) - Tubarão (SC), Brasil

Acadêmico do curso de medicina da Universidade do Sul de Santa Catarina (Unisul) - Tubarão (SC), Brasil.

Acadêmico do curso de medicina da Universidade do Sul de Santa Catarina (Unisul) - Tubarão (SC), Brasil 


\section{INTRODUÇÃO}

O câncer da pele é a neoplasia de maior incidência em várias regiões do mundo, ${ }^{1-4}$ inclusive no Brasil. ${ }^{1}$ O Instituto Nacional de Câncer (Inca) estimou, para o ano de 2006, 116.640 novos casos de câncer da pele não melanoma e 5.570 novos casos de melanoma. Somados, esses números correspondem a $26 \%$ do total dos casos novos de neoplasias malignas esperados no país. ${ }^{5}$ Esses dados refletem uma tendência mundial de aumento na incidência de carcinoma de células escamosas (CEC) da pele nas últimas décadas., ${ }^{3,-11}$

Na região sul do país, a taxa de câncer da pele é superior à nacional, sendo grande parte dela devida às taxas encontradas em Santa Catarina (SC). A maior concentração de câncer da pele no Brasil encontra-se neste estado, com estimativa de 125,78 casos por 100.000 habitantes para câncer não melanoma e 8,58 casos por 100.000 habitantes para melanoma. ${ }^{5}$

Os tumores malignos da pele são divididos em dois grandes grupos: melanoma e não melanoma. ${ }^{6,8}$ Neste último, incluem-se os carcinomas basocelular e de células escamosas, que juntos representam 95\% dos tumores malignos da pele. ${ }^{8,11}$ Sua incidência isolada é superior à dos carcinomas de pulmão, cólon, mama e reto e dos linfomas, somados. ${ }^{11}$

O CEC da pele aparece como a segunda neoplasia maligna da pele mais comum, representando $20 \%$ dos casos de câncer de pele no mundo. ${ }^{12,13}$

A histopatologia do CEC da pele consiste na proliferação e diferenciação atípicas de células espinhosas de caráter invasivo, ${ }^{13}$ o que o torna mais agressivo que o basocelular, tanto localmente quanto na capacidade de produzir metástases. ${ }^{13,14}$

Somente pequena porcentagem do CEC da pele primário é refratário ao tratamento dermatológico padrão, que consiste na excisão completa da lesão. Mesmo que de fácil tratamento em fase inicial e de rara mortalidade, as consequências do CEC da pele não tratado ou tardiamente diagnosticado têm importante impacto na saúde pública e individual. ${ }^{1,8,11,12,14,15} \mathrm{O}$ custo elevado do tratamento e a deterioração da qualidade de vida, em decorrência de sequelas psicológicas e físicas, denotam a importância do diagnóstico precoce. ${ }^{1}$

Apesar de existirem fatores de risco ainda desconhecidos para seu desenvolvimento, há unanimidade entre diversos estudos epidemiológicos de que existe associação significativa entre a exposição solar crônica e o desenvolvimento dessa neoplasia. ${ }^{1,3,4,8,12,13,16}$ Consequentemente, o CEC da pele é mais comum em idosos, resultado de radiação solar cumulativa. ${ }^{6,12,16}$

A maioria dos CECs de pele provém da evolução de ceratose actínica. ${ }^{4,13}$ Esta é caracterizada por lesões geralmente eritematosas, eventualmente acas- tanhadas, de caráter ceratótico e com escamas aderentes. Dor no local da lesão e infiltração celular demonstrável no exame histopatológico predizem maior possibilidade de transformação para carcinoma. ${ }^{13}$

É considerada fator de risco adicional para o desenvolvimento desse tipo de câncer da pele a presença de fenótipos desfavoráveis, ${ }^{1,13,16}$ representados pelos tipos de pele I e II (segundo a classificação de Fitzpatrick), ${ }^{12,13}$ cor clara dos olhos e presença de sardas e nevos. ${ }^{13}$ Além do fenótipo, mais dois fatores de risco são descritos: a história familiar de câncer da pele e a exposição ocupacional. ${ }^{3,13}$ Outros fatores de risco de menor importância, porém reconhecidamente carcinogênicos, são o alcatrão, o arsênico e a radioterapia. $^{1,13}$

Segundo a literatura, a incidência do carcinoma de células escamosas da pele é maior no gênero masculino, ${ }^{2,4-10,12,16-18}$ podendo afetar os homens até duas vezes mais que as mulheres. Essa estatística está possivelmente relacionada a atividades ocupacionais com exposição solar, geralmente predominantes no gênero masculino. ${ }^{3,6}$ Por motivo semelhante, a maioria dos CECs da pele são encontrados na cabeça (face e pescoço), sendo esses locais de grande exposição solar. $^{2,4,7,12-14,17}$

Como mencionado anteriormente, o estado de SC apresenta a maior incidência de câncer da pele no Brasil, ${ }^{5}$ porém não há dados consistentes na literatura que esclareçam as razões para esse fato, tampouco se é crescente como no restante do país.

A combinação de dois fatores possivelmente explica essa condição: a população catarinense é composta principalmente de indivíduos com ascendência europeia, que proporcionam as características fenotípicas supracitadas, ${ }^{1,12}$ e estaria exposta a grande intensidade de radiação ultravioleta, decorrente do comprometimento da camada de ozônio nesse estado. ${ }^{1,12,19}$

A elevada frequência do CEC da pele no país, principalmente em SC, constitui importante problema de saúde pública. ${ }^{1,8,12,14,15} \mathrm{Em}$ posse do conhecimento da epidemiologia dessa neoplasia, acredita-se que haveria maior valorização e incremento na suspeição diagnóstica por parte de médicos e outros profissionais da saúde. Partindo da premissa de que se conhece a estimativa de CEC da pele no estado de SC, mas ainda não se determinou se sua incidência é crescente, ou se essa estimativa é válida, este estudo procura esclarecer essas dúvidas, além de estabelecer o perfil epidemiológico dos pacientes dessa neoplasia entre os habitantes da cidade de Tubarão (SC).

\section{MÉTODOS}

Foi realizado estudo epidemiológico do tipo observacional com delineamento transversal, baseado 
na análise de laudos anatomopatológicos.

Foram analisados todos os laudos positivos para CEC da pele diagnosticados por exame anatomopatológico na cidade de Tubarão (SC), e por seleção aleatória sistemática, com intervalo de dois anos entre as amostras, foram escolhidos os anos de 2000, 2003 e 2006.

Foram incluídos todos os laudos anatomopatológicos com resultados positivos para CEC invasivo ou in situ, bem como os sinônimos carcinoma epidermoide, espinocelular, escamocelular, e formas especiais como a doença de Bowen e o carcinoma verrucoso da pele de pacientes residentes em Tubarão (SC), no período em que o diagnóstico foi obtido. Foram excluídos os laudos com diagnósticos histológicos diferentes de CEC da pele e de pacientes que não residiam em Tubarão (SC).

A fonte de dados utilizada foi secundária, pesquisada diretamente nos bancos de dados que continham os exames anatomopatológicos armazenados nos computadores dos laboratórios São Lucas e DiPrever, que são os únicos laboratórios na cidade de Tubarão (SC) que realizam tal exame.

$\mathrm{O}$ instrumento de coleta dos dados foi constituído de um protocolo de registro de informações. A coleta dos dados foi realizada no período de dezembro de 2007 a maio de 2008.

Foram analisadas as seguintes variáveis: ano da confirmação anatomopatológica, idade do paciente, gênero, maior diâmetro da lesão e localização da lesão. A variável populacional foi extraída do Departamento de Informática do Sistema Único de Saúde (Datasus). ${ }^{20}$

Para análise estatística, os dados foram digitados utilizando-se o programa Epidata ${ }^{\circledR} 3.1$ e analisados com auxílio do programa Epiinfo ${ }^{\circledR}$ 6.04. O cálculo da incidência foi realizado referente a 100,000 habitantes/ano, utilizando o número de neoplasias encontradas e os dados populacionais do Datasus20 para os anos escolhidos, como realizado em estudos semelhantes e nas estimativas para câncer do Inca. ${ }^{5,12}$ As frequências das variáveis foram descritas em valores absolutos e relativos.

O projeto deste trabalho foi enviado para avaliação da comissão de ética em pesquisa (CEP-Unisul) e, após aprovação, a coleta referente ao tema do trabalho foi efetuada da forma descrita.

\section{RESULTADOS}

Foram encontrados 200 laudos positivos para CEC da pele nos laboratórios anatomopatológicos do município de Tubarão (SC). Dos laudos positivos para CEC de pele, 103 (51,5\%) eram de pacientes do gênero feminino.

A incidência por 100.000 habitantes, segundo ano de ocorrência, foi de 94,39 para o ano de 2006 , de 71,16 para o ano de 2003 e de 50,86 para o ano de 2000. Nota-se aumento de $40 \%$ no risco de adquirir CEC de pele quando comparados os anos de 2000 e 2003 , e aumento de $86 \%$ quando comparados os anos de 2000 e 2006. A crescente incidência pode ser observada no gráfico 1 .

Embora no ano 2000 houvesse incidência maior de CEC da pele no gênero feminino, nos anos de 2003 e 2006 foi verificada maior incidência no gênero masculino. A incidência por 100.000 habitantes de CEC da pele para ambos os gêneros é demonstrada no gráfico 2 .

A idade variou de 28 a 96 anos, com média de 65,6 anos, mediana de 67 anos e moda de 72 anos. A distribuição por faixa etária mostrou maior número de casos tanto no gênero masculino - 60 casos (66,6\%) - quanto no feminino - 71 casos $(71,7 \%)-$ em pacientes acima de 60 anos (Tabela 1).

$\mathrm{Na}$ tabela 2, verifica-se a distribuição dos CECs de pele conforme a localização da lesão. Em ambos os gêneros, o local de ocorrência mais comum foi face 44 casos -, correspondendo a 57,1\% dos 77 laudos que informavam esse dado. Entretanto, o gênero masculino apresentou risco $60 \%$ maior de ocorrência de lesão na face quando comparado ao gênero feminino.

Quando analisadas as porcentagens de CEC de pele de acordo com o subtipo histológico encontrado no estudo, foram mais frequentes os subtipos in situ e bem diferenciado, com 164 casos $(83,0 \%)$, em seguida o moderadamente diferenciado, com 29 (14,7\%), e o menos frequente, o pouco diferenciado, somando apenas quatro (2,0\%) dos casos estudados. Três laudos positivos para CEC de pele não evidenciaram essa informação.

O maior diâmetro das lesões encontradas nesse

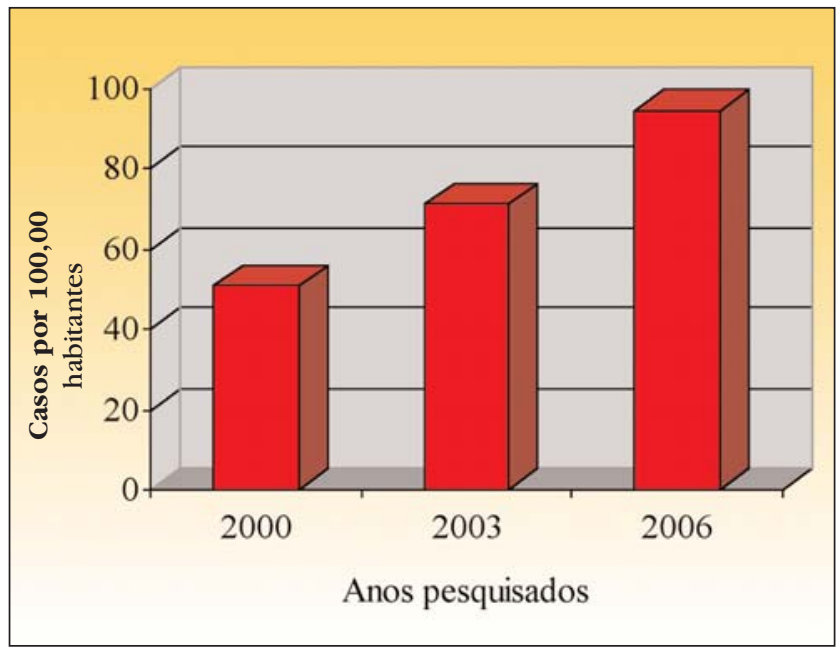

Gráfico 1: Incidência de carcinoma de células escamosas da pele, por 100.000 habitantes, em Tubarão (SC) nos anos 2000, 2003 e 2006 


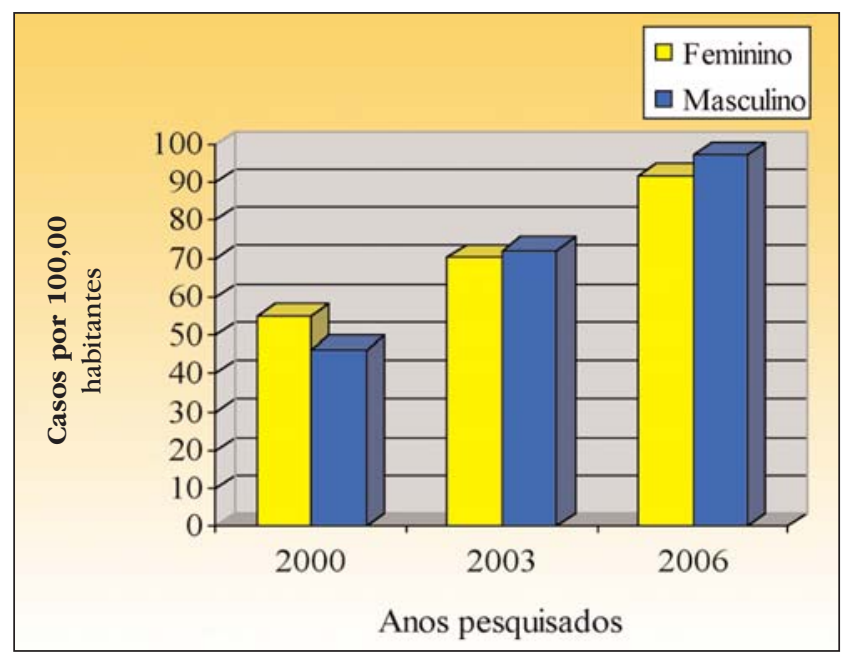

Gráfico 2: Incidência de carcinoma de células escamosas da pele, por 100.000 habitantes, conforme o gênero, em Tubarão (SC) nos anos 2000, 2003 e 2006

estudo variou de 0,3-10 cm; a média foi de $1,9 \mathrm{~cm}$, e a mediana e a moda, de $1,5 \mathrm{~cm}$. Nota-se, na tabela 3 , que apenas nove $(4,57 \%)$ das lesões biopsiadas apresentaram maior diâmetro $\geq 4 \mathrm{~cm}$.

\section{DISCUSSÃO}

Dados de alta qualidade sobre a incidência de CEC da pele são raros na literatura, pois frequentemente os registros de câncer excluem cânceres de pele não melanoma ou proporcionam dados incompletos em relação a suas características. ${ }^{8,9,17,21}$ Nos últimos anos, pelo acesso a bancos de dados eletrônicos, os registros de CEC da pele confirmado por exame anatomopatológico tem sido mais bem definido.

Utilizando os bancos de dados dos laboratórios que realizam exames anatomopatológicos na cidade de Tubarão (SC), foi possível incluir neste estudo a totalidade dos casos registrados de CEC da pele nos anos estudados. Portanto, é provável que os resultados desta pesquisa se aproximem mais que estimativas passadas da incidência dessa neoplasia neste município.

O levantamento dos laudos histopatológicos positivos para CEC da pele na cidade de Tubarão (SC) mostrou incidência elevada e crescente. Uma vez que não existe informação específica do Inca para o CEC da pele, foram utilizados os dados fornecidos para cânceres de pele não melanoma. Para possibilitar a realização de comparações entre os dados estimados pelo Inca e os dados encontrados neste estudo, foi necessário saber qual a porcentagem de câncer da pele não melanoma que pode ser atribuída ao CEC da pele. Uma revisão da literatura consultada revelou o CEC da pele como responsável por aproximadamente $20 \%$ de todos os cânceres de pele não melanoma. ${ }^{12,13}$
Com essas informações, estimaram-se as previsões de incidência de CEC da pele, de acordo com o Inca, para os anos estudados e, assim, procedeu-se às comparações desejadas.

Os resultados encontrados nesta pesquisa ultrapassaram, em quantidade considerável, a incidência esperada para os anos pesquisados, de forma que as estimativas de CEC da pele para o ano de 2006 foram de 25,16 por 100,000 habitantes no gênero masculino e 23,50 por 100.000 habitantes no gênero feminino, e as encontradas neste estudo foram de 95,00 e 91,78 por 100.000 habitantes nos gêneros masculino e feminino, respectivamente.

As incidências não separadas por gênero, encontradas no atual estudo, também ultrapassam os valores encontrados em estudo semelhante realizado em Blumenau (SC) nos anos de 1980 a 1999. Nesse estudo, foi constatado coeficiente de morbidade de 43,8 por 100.000 habitantes no ano de $1999^{12}$ - coeficiente menor, porém não discrepante em relação à incidência de 50,86 por 100.000 habitantes encontrada no atual estudo no ano de 2000. Entretanto, quando comparados os resultados do estudo com as incidências dos anos de 2003 e 2006, respectivamente 71,16 e 94,3 por 100.000 habitantes, são verificadas diferenças consideráveis. É importante salientar que o estudo de Blumenau, diferente do atual, incluiu em sua casuística o CEC de lábio. ${ }^{12}$

As incidências encontradas, destoantes dos dados nacionais, parecem ser válidas quando comparadas aos estudos realizados em países como Estados Unidos e Austrália. Nesses países, encontram-se índices de incidência de alta qualidade, mesmo não havendo método de notificação compulsória. Os dados de incidência são frequentemente estratificados em trabalhos patrocinados por seus governos e utilizam estudos populacionais e prontuários médicos, além de confirmações anatomopatológicas. Esses países têm reconhecido o câncer da pele como problema de saúde pública nacional nas últimas décadas.

Na Austrália, onde é encontrada a maior incidência de CEC da pele no mundo, também é observado o maior número de pesquisas a respeito dessa neoplasia. Em um estudo realizado no ano de 2002, foi estimada incidência de 387 casos de CEC da pele para cada 100.000 habitantes, bem como aumento crescente na sua incidência, quando analisados os dados registrados desde o ano de $1985 .{ }^{16}$

Nos Estados Unidos, referente ao ano de 1994, foi encontrada incidência por 100.000 habitantes de 81 a 136 casos para o gênero masculino e 26 a 59 para o gênero feminino. ${ }^{22}$ Provavelmente a incidência atual, acompanhando a tendência mundial, encontre-se ainda maior. 
Tabela 1: Distribuição da frequência e percentual dos casos de carcinoma de células escamosas da pele, de acordo com idade e gênero, em Tubarão (SC) nos anos de 2000, 2003 e 2006

\begin{tabular}{lllllll}
\hline Idade em anos & \multicolumn{2}{l}{ Frequência } & \% Masculino & \multicolumn{2}{c}{ Frequência \% Feminino } & \multicolumn{2}{c}{ Frequência (\%) Geral } \\
\hline $0-39$ & 8 & 8,9 & 4 & 4,0 & 12 & 6,3 \\
$40-49$ & 7 & 7,8 & 11 & 11,1 & 18 & 9,5 \\
$50-59$ & 15 & 16,7 & 13 & 13,1 & 28 & 14,8 \\
$60-69$ & 27 & 30,0 & 23 & 23,2 & 50 & 26,5 \\
$70-79$ & 22 & 24,4 & 28 & 28,3 & 50 & 26,5 \\
$80-89$ & 7 & 7,8 & 18 & 18,2 & 25 & 13,2 \\
$>90$ & 4 & 4,4 & 2 & 2,0 & 6 & 3,2 \\
Não informada & - & & - & & 11 & \\
\hline
\end{tabular}

O aumento na incidência do CEC da pele, nos últimos anos, pode ser atribuído a diversas condições observadas na população estudada.

A primeira, e provavelmente a mais importante, é o aumento na radiação ultravioleta decorrente da depleção na camada de ozônio. ${ }^{1,3,6,12,19}$ É previsto que, para cada $10 \%$ de perda da camada de ozônio, haja aumento de $40 \%$ na incidência de câncer da pele. ${ }^{23}$ Sendo assim, uma depleção de pequena quantidade pode influenciar a incidência do câncer da pele em proporção considerável.

De acordo com dados meteorológicos, na região do estado de SC houve diminuição de 7 a $8 \%$ da camada de ozônio, por década, desde 1979 até $2000,{ }^{19}$ influenciando inevitavelmente a incidência encontrada neste estudo. Além disso, a maior parte da população de SC tem cor da pele clara, decorrente de colonização europeia. ${ }^{1,12}$ Esse tipo de pele é mais susceptível aos danos causados pelo aumento da radiação ultravioleta, tendo maior tendência a desenvolver lesões neoplásicas quando exposto cronicamente a esses raios. ${ }^{1,8,12,16}$

A segunda condição deflagradora dessa alta incidência é a educação e conscientização dos médicos e principalmente da população em geral, o que pode ter influenciado positivamente o aumento na detecção dos tumores, que antigamente passariam despercebidos. . $^{2,3,8}$
No Brasil, programas e campanhas de prevenção do câncer da pele se iniciaram há aproximadamente 10 anos. As campanhas têm como objetivo a prevenção primária - proteção contra a exposição à luz solar - e secundária, realizando diagnóstico precoce e tratamento oportuno. ${ }^{1}$

Indivíduos hoje acima de 60 anos, responsáveis por mais de $50 \%$ da incidência de CEC da pele, ${ }^{12,17}$ foram, no máximo, alvos de prevenção secundária dessas campanhas. Sendo assim, até então é plausível considerar que os benefícios dessas campanhas estejam refletidos inversamente nos dados de incidência da população. Provavelmente a melhora na capacidade dos médicos e profissionais da saúde em rastrear esse tumor e a maior conscientização da população sejam parcialmente responsáveis pelo aumento da sua incidência.

Além disso, há indiscutível envelhecimento populacional ocorrendo em diversas partes do mundo, inclusive no Brasil. De acordo com dados do Instituto Brasileiro de Geografia e Estatística, no ano de 1991 idosos acima de 60 anos representavam $6,75 \%$ da população de SC e no ano de 2000 foram responsáveis por $8,03 \% .{ }^{24} \mathrm{~A}$ exposição cumulativa a raios ultravioleta está fortemente associada à alta incidência de CEC da pele, sendo este, em comparação com os outros dois cânceres de pele prevalentes, $o$

Tabela 2: Frequência e porcentagem dos casos de carcinoma de células escamosas da pele, de acordo com localização e gênero, em Tubarão (SC) nos anos de 2000, 2003 e 2006

\begin{tabular}{lllllll}
\hline Localização da lesão & Frequência & \% Masculino & Frequência & \% Feminino & Frequência & \% Geral \\
\hline Face & 33 & 66 & 11 & 25 & 44 & 57,1 \\
Membro superior & 8 & 16 & 9 & 33,3 & 17 & 22,1 \\
Membro inferior & 6 & 12 & 7 & 25,9 & 13 & 16,9 \\
Tronco & 3 & 6 & 0 & 0,0 & 3 & 3,9 \\
Não informada & - & & - & & 123 & \\
\hline
\end{tabular}


TABela 3: Frequência e porcentagem dos casos de carcinoma de células escamosas da pele, conforme o maior diâmetro da lesão, em Tubarão (SC) nos anos de 2000, 2003 e 2006

\begin{tabular}{lll}
\hline Maior diâmetro $(\mathbf{c m})$ & Frequência & \% \\
\hline $0-0,5$ & 6 & 3,04 \\
$0,6-1$ & 43 & 12,83 \\
$1,1-1,5$ & 54 & 27,41 \\
$1,6-2,0$ & 35 & 17,77 \\
$2,1-2,5$ & 20 & 10,15 \\
$2,6-3,0$ & 16 & 8,12 \\
$3,1-3,5$ & 7 & 3,55 \\
$3,6-4,0$ & 5 & 2,54 \\
$4,1-4,5$ & 3 & 1,52 \\
$>4,5$ & 6 & 3,05 \\
Não informado & 5 & \\
\hline
\end{tabular}

mais associado com pacientes idosos, ${ }^{6,12,16}$ de forma que, havendo aumento da população idosa, é esperado também incremento na incidência de CEC da pele.

Por fim, a mudança no vestuário e no estilo de vida, acarretando maior exposição ao sol, também tem sido discutida como fator agravante no aumento da incidência de CEC da pele. ${ }^{1,3,6,8}$ Tem-se tornado frequente o hábito de exposição solar com objetivo de bronzeamento da pele, que é de alto valor estético no país. ${ }^{1,3}$

A proporção dos gêneros de aproximadamente 1:1, encontrado no atual estudo, é inédita na literatura. Estudos prévios revelaram o gênero masculino como mais incidente, ${ }^{2,4-10,12,16-18}$ em geral na proporção gênero masculino para feminino de $2: 1$. Esse fato pode ser atribuído à maior procura de atendimento médico pelo gênero feminino, já que, como mencionado anteriormente, os registros que fornecem os valores de incidência dependem diretamente da procura médica. Evidenciou-se em campanhas de prevenção que, entre os examinados, a proporção de pacientes representada pelo gênero masculino não atingiu $40 \%{ }^{1}$

Também é importante salientar que mesmo não havendo equilíbrio na proporção de gênero na incidência de CEC da pele na literatura revisada, existem vários estudos que revelam aumento maior na incidência de câncer da pele no gênero feminino em comparação ao gênero masculino., ${ }^{2,6,18,21}$

Pelos motivos já descritos, a maior porcentagem de incidência de CEC da pele é encontrada na população acima de 60 anos. ${ }^{4,12,17}$ Há outros estudos que mostram que a média de idade de diagnóstico foi maior que 65 anos. ${ }^{7,10,21}$ Neste estudo foi encontrada maior quantidade de casos, 131 (69,4\%), em pacientes acima de 60 anos e com idade média de diagnóstico de 65,6 anos, similar aos números encontrados na literatura acima citada.

O local mais frequente para a ocorrência do CEC da pele, em ambos os gêneros, foi a região da cabeça, mais especificamente a face, concordando com a maior parte da literatura. , $, 4,12,13,14,17^{\text {Esse achado }}$ reforça a importância da exposição solar crônica como fator de risco, sendo a face local bastante exposto ao sol no dia-a-dia.

Além disso, constatou-se que os homens têm risco $60 \%$ maior que as mulheres de o CEC da pele ocorrer na face. Um dos motivos que corroboram esse achado é a exposição solar ocupacional, mais comum no gênero masculino. ${ }^{3,6}$ Outra hipótese seria a maior preocupação do gênero feminino com a estética e os cuidados com a pele. ${ }^{1,3}$

A média do maior diâmetro da lesão, no atual estudo, foi de $1,9 \mathrm{~cm}$, similar à de outro estudo, cujo diâmetro médio foi de $2 \mathrm{~cm}$. No mesmo estudo ficou estabelecido que lesões apresentando maior diâmetro de $4 \mathrm{~cm}$ ou mais se relacionavam com potencialidade maior de invasão tecidual.14 Uma vez que no atual estudo somente nove $(4,57 \%)$ das lesões encontradas apresentavam tais diâmetros, é possível especular que a maioria das lesões causadas pelo CEC da pele, no município de Tubarão (SC), tenham sido de baixa potencialidade de invasão.

\section{CONCLUSÃO}

Foi possível traçar um perfil do paciente portador do CEC da pele e da situação atual dessa neoplasia na população de Tubarão (SC).

1. Não houve predomínio importante de gênero quanto à incidência;

2. A maior incidência foi encontrada nos pacientes acima de 60 anos de idade;

3. Há predominância importante de lesões na face;

4. A maioria das lesões, 186 (95,43\%), tinha seu maior diâmetro menor que $4 \mathrm{~cm}$.

A incidência de casos de CEC da pele, confirmados por exames anatomopatológicos, é maior que a previamente estimada. A distribuição dos casos mostrou aumento crescente da incidência entre os anos de 2000, 2003 e 2006.

Conhecer o aumento da incidência dessa enfermidade e o perfil de seu portador nos possibilita planejar melhor as ações no campo da educação sanitária e da prevenção primária e secundária dessa neoplasia. 


\section{REFERÊNCIAS}

1. Sociedade Brasileira de Dermatologia. Análise de dados das campanhas de prevenção ao câncer de pele promovidas pela Sociedade Brasileira de Dermatologia de 1999 a 2005. An Bras Dermatol. 2006;81:533-9.

2. Athas WF, Hunt WC, Key CR. Changes in nonmelanoma skin cancer incidence between 1977-1978 and 19981999 in Northcentral New Mexico. Cancer Epidemiol Biomarkers Prev. 2003;12:1105-8.

3. Hora C, Batista CVC, Guimarães PB, Siqueira R, Martins S. Avaliação do conhecimento quanto a prevenção do câncer de pele e sua relação com exposição solar em freqüentadores de academia de ginástica em Recife. An Bras Dermatol. 2003;79:693-701.

4. Dergham AP, Muraro CC, Ramos EA, Mesquita LAF, Collaço LM. Distribuição dos diagnósticos de lesões pré-neoplásicas e neoplásicas de pele no Hospital Universitário Evangélico de Curitiba. An Bras Dermatol. 2004;79:365-96.

5. INCA - Instituto Nacional de Câncer [homepage] Estimativa 2006: incidência de câncer no Brasil. [acesso 12 Out 2007]. Disponível em: http://www.inca.gov.estimativa/2006/.

6. de Vries E, van de Poll-Franse LV, Louwman WJ, de Gruijl FR, Coebergh JW. Predictions of skin cancer incidence in the Netherlands up to 2015. Br J Dermatol. 2005;152:481-8.

7. Katalinic A, Kunze U, Schäfer T. Epidemiology of cutaneous melanoma and non-melanoma skin cancer in Schleswig-Holstein, Germany: incidence, clinical subtypes, tumour stages and localization (epidemiology of skin cancer). Br J Dermatol. 2003;149:1200-6.

8. Estrada JG. Non-melanoma skin cancer in the Mediterranean area. Eur J Dermatol. 2007;44:922-4

9. Hoey SE, Devereux CE, Murray L, Catney D, Gavin A, Kumar S, et al. Skin cancer trends in Northern Ireland and consequences for provision of dermatology services. Br J Dermatol. 2007;156:1301-7.

10. Holme SA, Malinovszky K, Roberts DL. Changing trends in non-melanoma skin cancer in South Wales, 1988-98. Br J Dermatol. 2000;143:1224-9.

11. Rocha FP, Menezes AMB, Almeida JHL, Tomasi E. Especificidade e sensibilidade de rastreamento para lesões cutâneas pré-malignas e malignas. Rev Saúde Pública. 2002;36:101-6.

12. Nasser N. Epidemiologia dos cânceres espinocelulares - Blumenau (SC) - Brasil, de 1980 a 1999. An Bras Dermatol. 2004;80:363-8

13. Amaral ACN, Azulay RD, Azulay DR. Neoplasias epiteliais. In: Azulay RD, Azulay DR, editores. Dermatologia. 4 ed. Rio de Janeiro: Guanabara Koogan S.A.; 2006. p. 510-26.

14. Clayman GL, Lee JJ, Holsinger FC, Zhou X, Duvic M, El-Naggar AK, et al. Mortality risk from squamous cell skin cancer. J Clin Oncol. 2005;23:759-65.
15. Housman TS, Feldman SR, Williford PM, Fleischer AB Jr, Goldman ND, Acostamadiedo JM, et al. Skin cancer is among the most costly of all cancers to treat for the Medicare population. J Am Acad Dermatol. 2003; 48:425-9.

16. Staples MP, Elwood M, Burton RC, Williams JL, Marks R, Giles GG. Non-melanoma skin cancer in Australia: the 2002 national survey and trends since 1985. Med J Aust. 2006;184:6-10.

17. Brewster DH, Bhatti LA, Inglis JHC, Nairn ER, Doherty VR. Recent trends in incidence of nonmelanoma skin cancers in the East of Scotland, 1992-2003. Br J Dermatol. 2007; 156:1295-300.

18. Glass AG, Hoover RN. The emerging epidemic of melanoma and squamous cell skin cancer. JAMA. 1989;262:2097-100.

19. Scientific Assessment of Ozone Depletion: 2002. World Meteorological Organization. Global Ozone Research and Monitoring Project - Report n. 47 [homepage on the Internet]. [acesso 7 Mai 2008].Disponível em: http://www.esrl.noaa.gov/csd/assessments/2002/ executive summary.html.

20. DATASUS - Departamento de Informática do SUS População Residente - Estimativa para o TCU - Santa Catarina. [acesso 20 Jan 2008]. Disponível em: tabnet.datasus.gov.br/cgi/deftohtm.exe?ibge/cnv/poptsc.def.

21. Stang A, Ziegler S, Büchner U, Ziegler B, Jöckel KH, Ziegler V. Malignant melanoma and nonmelanoma skin cancers in Northrhine-Westphalia, Germany: a patient- vs. diagnosis-based incidence approach. Int $\mathrm{J}$ Dermatol. 2007; 46:564-70.

22. Miller DL, Weinstock MA. Nonmelanoma skin cancer in the United States: incidence. J Am Acad Dermatol. 1994;30:774-8.

23. Oikarinen A, Raitio A. Melanoma and other skin cancer in circumpolar areas. Int $\mathrm{J}$ Circumpolar Health. 2000;59:52-6.

24. IBGE - Instituto Brasileiro de Geografia e Estatística Perfil dos idosos responsáveis pelos domicílios no Brasil 2000. [acesso 20 Jan 2008]. Disponível em: http:/www.ibge.gov.br/home/estatistica/populacao/ perfilidoso/tabela1_1.shtm.

ENDEREÇO PARA CORRESPONDÊNCIA / MAILING ADDRESS:
Prof. Daniel Holthausen Nunes
Rua Professor Herminio Jacques, 122
88015180 Florianópolis SC
Tel.:/FAx: $483224-2740$
E-mail: daniel@floripa.com.br

ENDEREÇO PARA CORRESPONDÊNCIA / MAILING ADDRESS:

Prof. Daniel Holthausen Nunes

Rua Professor Hermínio Jacques, 122

88015180 Florianópolis SC

E-mail:daniel@floripa.com.br

Como citar este artigo/How to cite this article: Nunes DH, Back L, Silva RV, Medeiros VS. Incidência do carcinoma de células escamosas da pele na cidade de Tubarão (SC) - Brasil nos anos de 2000, 2003 e 2006 . An Bras Dermatol. 2009;84(5):482-8. 